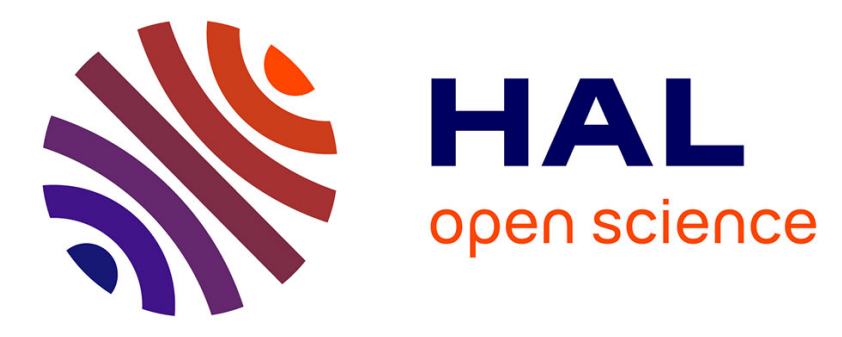

\title{
A computational procedure for the prediction of settlement in granular materials under cyclic loading
}

A. Karrech, Denis Duhamel, Guy Bonnet, Jean-Noël Roux, François Chevoir, Jean Canou, Jean Claude Dupla, Karam Sab

\section{- To cite this version:}

A. Karrech, Denis Duhamel, Guy Bonnet, Jean-Noël Roux, François Chevoir, et al.. A computational procedure for the prediction of settlement in granular materials under cyclic loading. Computer Methods in Applied Mechanics and Engineering, 2007, 197 (1-4), pp.80-94. 10.1016/j.cma.2007.07.011. hal-00691064

\section{HAL Id: hal-00691064 \\ https://hal.science/hal-00691064}

Submitted on 15 Jan 2016

HAL is a multi-disciplinary open access archive for the deposit and dissemination of scientific research documents, whether they are published or not. The documents may come from teaching and research institutions in France or abroad, or from public or private research centers.
L'archive ouverte pluridisciplinaire HAL, est destinée au dépôt et à la diffusion de documents scientifiques de niveau recherche, publiés ou non, émanant des établissements d'enseignement et de recherche français ou étrangers, des laboratoires publics ou privés. 


\title{
A computational procedure for the prediction of settlement in granular materials under cyclic loading
}

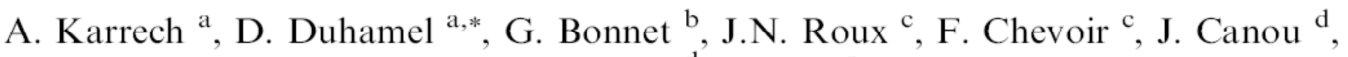 \\ J.C. Dupla ${ }^{\mathrm{d}}, \mathrm{K}$. Sab ${ }^{\mathrm{a}}$ \\ ${ }^{a}$ Laboratoire Analyse des Matériaux et Identification, Ecole Nationale des Ponts et Chaussées (ENPC), Institut Navier (IN), 6 et 8 Avenue Blaise Pascal, \\ Cité Descartes, Champs sur Marne, 77455 Marne-La-Vallée, Cedex 2, France \\ ${ }^{\mathrm{b}}$ Laboratoire de Mécanique, Université de Marne-La-Vallée, France \\ ${ }^{\mathrm{c}}$ Laboratoire des Matériaux et Structures du Génie Civil, ENPC, IN, France \\ ${ }^{\mathrm{d}}$ Centre d'Enseignement et de Recherche en Mécanique des Sols, ENPC, IN, France
}

Abstract

Granular materials have been subject of considerable interest in the recent years because of the richness of their physical behavior. Coupled with experimental approaches, the discrete element methods are now recognized as powerful tools to understand several mechanisms related to these materials such as convection, compaction and settlement. However, these methods are very costly in terms of calculation time.

In this paper, we introduce a computational method for the simulation of three-dimensional granular bed responses under long term cyclic loading. It sequentially uses a molecular dynamics scheme, a time averaging technique, and a relaxation method in order to predict the long term flow. The suggested approach is then applied to specific cases in order to verify its efficiency and accuracy.

(c) 2007 Elsevier B.V. All rights reserved.

Keywords: Settlement; Granular materials; Molecular dynamics; Time averaging; Relaxation

\section{Introduction}

Granular materials have been found to be very useful for road pavements and railway track platforms, because of their porosity, vibrational characteristics and rigidity. However, with the increase in vehicle speeds and high comfort standards, a better understanding of granular behavior under dynamic loading is required. Therefore, accurate prediction of local long term residual displacements and induced forces is necessary in assessing the capacity of infrastructures and preventing their differential settlement. These types of materials can exhibit astonishing and roughly understood phenomena under vibration. Several experimental devices and numerical methods were devel-

\footnotetext{
" Corresponding author. Tel.: +331641537 28; fax: +33164153741.
} E-mail address: duhamel@lami.enpc.fr (D. Duhamel). oped in order to investigate the behavior of granular materials subjected to cyclic loading. In the early seventies Shenton [1] studied granular residual deformations under repeated loading using triaxial testing. He obtained an empirical logarithmic law which describes granular settlement with respect to the number of cycles. Guérin [2] developed an experimental setup and studied the settlement of dimensionally reduced ballast samples. Results showed the existence of two settlement phases, the first one takes place at the beginning of the process and corresponds to the compaction regime whereas the second takes place beyond a relatively high number of cycles; it corresponds to the stabilized regime. Testing results showed that the second regime can be described with a power law relating the elastic deflexion to the settlement rate with respect to the number of cycles. Later on, Bodin [3] studied the same phenomenon under vertical and lateral excitations, she 
ended up with a similar empirical law which includes a coupling effect between lateral and vertical permanent displacements. More recently, Al-Shaer [4] produced a dimensionally reduced railway platform, on which he studied the granular settlement in the vertical direction for different train speeds. The obtained results show that the settlement speed increases non-linearly with respect to the acceleration intensity. In addition, the power laws obtained by Guérin [2] and Bodin [3] at relatively low acceleration intensities (accelerations lower than the $10 \mathrm{~ms}^{-2}$ ) are no longer valid.

In general, experimental tests provide reliable results that can describe the global behavior of granular materials. However, in many cases experiments are limited in terms of number of geometrical and mechanical parameters. In addition, without a high degree of sophistication, the existing experimental setups do not allow access to local stress, displacement and acceleration fields, thereby, they jeopardize the understanding of the granular behavior. Numerical methods can represent trustworthy complements. One of the recent interesting models specially developed for granular materials was suggested by Nguyen [5]. The particularity of this phenomenological continuum model lies in its unidirectional aspect. The non-tension effects observed in granular materials are considered when developing the constitutive law. More recently, Abdelkrim [6] suggested a computational step by step algorithm which uses a cyclic constitutive law relating the progressive accumulation of irreversible displacement to the cyclic stress generated by the traffic loading, applied it to the granular materials considered as a continuum elastic medium, and predicted the permanent displacement of the following step. When repeated for a large number of cycles, this procedure can produce interesting results in a relatively limited number of iterations. However, this algorithm assumes the existence of residual stresses under repeated loads. This assumption can be relaxed in case of discrete element analysis as permanent deformations are not necessarily related to permanent stresses, but can be attributed to the granular rearrangement, and migration under repeated loading. Saussine et al. [7] used a full contact dynamic to describe $2 \mathrm{D}$ polygonal granular materials settlement under repeated loading and showed an interesting agreement between the discrete element approach and the experimental results.

According to the above mentioned studies, granular materials settlement under repeated loading can be due to some or each one of the following issues: (i) material compaction due to the cyclic loading, (ii) migration and flow of granular particles to less loaded zones, and (iii) granular overwork and failure, (iv) lateral and vertical loading coupling effect $[8,9]$. In this paper, we focus only on the first two issues. It is assumed that the ground/granular interface overlapping is negligible as compared to the permanent deformation. The granular particles are assumed to be rigid with elastic contacts and the excitation is taken as purely vertical. With the advent of discrete element methods, it is possible to simulate complex flows of granular materials and to cover a high range of parameters. Besides, unlike the continuum models which rely on homogenization techniques [10], by definition discrete methods allow independent particles flow. Therefore, granular rearrangements can be observed and quantified in order to evaluate their effect in the settlement mechanism.

Since its introduction by Cundall and Strack [11] for mechanical engineering applications, the molecular dynamics (MD) method is gaining momentum and becoming an interesting computational tool for granular materials analysis. Associated with appropriate time averaging and relaxation technique, the molecular dynamics represents an interesting mean for studying the settlement mechanism under dynamic loading, in more detail than the experimental tests would usually allow.

The present paper, therefore, introduces a computational method which associates a molecular dynamics scheme, the engine of the suggested method, with time averaging and relaxation techniques, in order to describe the granular settlement under dynamic loading. In Section 2 , the molecular dynamics scheme adopted for this study is briefly introduced. In addition, a particular interest is given to the contact models which govern the granular interactions. In Section 3, the estimation technique is explained and tested in terms of agreement with full calculations. In Section 4, the relaxation method is derived and explained through two simple examples. Finally, the whole procedure is presented in a sequential flow chart in Section 5, moreover, results regarding the convergence of the suggested method and settlement mechanisms are presented for different field parameters.

\section{Discrete element simulation model}

Consider a system $\mathscr{S}$ of $N$ isotropic rigid particles with elastic contacts, equal mechanical characteristics, randomly distributed diameters $d_{\beta \in[1, N]}$. These particles are placed into a cylindrical container of diameter $D$. The ratio $D / d$ is high enough to avoid any size effects. In our simulations, this parameter is about 25 , unless differently indicated. The container is open from the top in order to apply the appropriate boundary conditions according to the desired modes of vibration.

\subsection{Equations of motion}

The molecular dynamics, as adopted by the mechanical engineering researchers [11-13] consist of modeling the granular motion using Newton equations, in order to describe the flow. For a given particle, $\beta$ of mass $m_{\beta}$ and moment of inertia $I_{\beta}$, the equation of motion can be written as follows:

$$
\left\{\begin{array}{l}
F_{\beta}+\sum_{\alpha \neq \beta \in \mathscr{S}} F_{\alpha \beta}=m_{\beta} \ddot{r}_{\beta}, \\
M_{\beta}+\sum_{\alpha \neq \beta \in \mathscr{S}} M_{\alpha \beta}=I_{\beta} \ddot{\theta}_{\beta},
\end{array}\right.
$$


where $\left(F_{\beta}, M_{\beta}\right)$ represent the external actions, $\left(F_{\alpha \beta}, M_{\alpha \beta}\right)$ denote the contact actions of a particle $\alpha$ on $\beta$, and $\left(\ddot{r}_{\beta}, \ddot{\theta}_{\beta}\right)$ are the particle accelerations. Several numerical finite difference schemes such as Verlet, leap-frog, or predictor-corrector algorithms can be used to integrate the above mentioned second-order equations of motion. The predictor-corrector algorithm is used herein. It consists of first predicting the displacements and velocities using a Taylor development:

$$
\begin{aligned}
& r_{\beta}^{p}(t+\Delta t)=r_{\beta}(t)+\dot{r}_{\beta}(t) \Delta t+\ddot{r}_{\beta}(t) \frac{\Delta t^{2}}{2}+\mathcal{O}\left((\Delta t)^{3}\right), \\
& i_{\beta}^{p}(t+\Delta t)=\dot{r}_{\beta}(t)+\ddot{r}_{\beta}(t) \Delta t+\mathcal{O}\left((\Delta t)^{2}\right) .
\end{aligned}
$$

The predicted configuration is used to calculate the contact interactions at $t+\Delta t$. The resulting accelerations are generally different than the predicted accelerations. The differences between them can be written as follows:

$\Delta a=\ddot{r}_{\beta}^{c}(t+\Delta t)-\ddot{r}_{\beta}^{p}(t+\Delta t)$.

The difference calculated in the above mentioned equation is used to correct the predicted fields. The new configuration as well as its corresponding velocities and accelerations read:

$$
\begin{aligned}
& r_{\beta}^{c}(t+\Delta t)=r_{\beta}^{p}(t+\Delta t)+c_{0} \frac{\Delta t^{2}}{2} \Delta a, \\
& \dot{r}_{\beta}^{c}(t+\Delta t)=i_{\beta}^{p}(t+\Delta t)+c_{1} \Delta t \Delta a, \\
& \ddot{r}_{\beta}^{c}(t+\Delta t)=\ddot{r}_{\beta}^{p}(t+\Delta t)+c_{2} \Delta a .
\end{aligned}
$$

According to Allen and Tildesley [14], the corrector coefficients are evaluated in order to optimize the algorithm convergence. In the case of three order predictor-corrector scheme, the coefficients are $c_{0}=0, c_{1}=1$ and $c_{2}=1$.

\subsection{Contact modeling}

Granular materials represent the assemblies of solid discrete particles. At the microstructural level, the granular behavior is mainly governed by the contact nature between the material constituents. Consequently, the description of the granular flow highly depends on the understanding of the granular interactions. The interactions considered herein are dissipative, non-linear, and loading path dependant. In the normal direction, Hertz-Mindlin law [15,16], is used to describe the elastic normal interactions:

$F_{\mathrm{n}}^{\mathrm{e}}=\frac{4}{3} E^{*} \sqrt{R^{*}} \delta_{\mathrm{n}}^{3 / 2}$.

The equivalent Young modulus between two particles $\alpha$ and $\beta$ is defined by $E^{*}=\frac{E}{2\left(1-v^{2}\right)}$, and the equivalent particle radius is defined by $\frac{1}{R^{*}}=\frac{1}{R_{x}}+\frac{1}{R_{\beta^{*}}}$. The Hertz law induces a circular contact surface of radius, a, which depends on the normal penetration $\delta_{\mathrm{n}}$. Assuming a uniform tangential displacement, uncoupled normal and tangential actions, and no slip at the contact interface, Johnson [17] showed that the tangential elastic contact force can be written as follows:

$F_{\mathrm{t}}^{\mathrm{e}}=8 G^{*} \sqrt{R^{*}} \delta_{\mathrm{n}}^{1 / 2} \delta_{\mathrm{t}}$.

The equivalent shear modulus between two particles $\alpha$ and $\beta$ is defined by $G^{*}=\frac{G}{2(2-v)}$. Note that the advantage of the above mentioned tangential contact law is its easy implementation as compared to more realistic but complex tangential contact models which are available in the literature [18]. The tangential displacement is evaluated using the relative tangential velocity of two overlapping particles since $t_{0}, \delta_{\mathrm{t}}=\int_{t_{0}}^{t} v_{t_{\alpha \beta}} \mathrm{d} t$. The normal and tangential forces acting on the particles also enclose viscous terms that depend on the mass and normal velocity. In addition, a coulomb friction is included through the threshold $F_{\mathrm{t}}=\mu F_{\mathrm{n}}$, where $\mu$ is the friction coefficient. Finally, the forces acting on a particle $\alpha$ can be written as follows:

$F_{\mathrm{n}}=F_{\mathrm{n}}^{\mathrm{e}}-\frac{m}{2} \gamma_{\mathrm{n}} v_{\mathrm{n}}$,

$F_{\mathrm{t}}= \begin{cases}F_{\mathrm{t}}^{\mathrm{e}}-\frac{m}{2} \gamma_{\mathrm{t}} v_{\mathrm{t}} & \text { if }\left\|F_{\mathrm{t}}\right\| \leqslant \mu F_{\mathrm{n}}, \\ \operatorname{sign}\left(F_{\mathrm{t}}^{\mathrm{e}}\right) \mu F_{\mathrm{n}}-\frac{m}{2} \gamma_{\mathrm{t}} v_{\mathrm{t}} & \text { if }\left\|F_{\mathrm{t}}\right\|>\mu F_{\mathrm{n}} .\end{cases}$

The phenomenological constant coefficients $\gamma_{n, t}$ describe the normal and tangential viscous damping. In case of linear contact, these coefficients are related to the restitution coefficients $e_{\mathrm{n}, \mathrm{t}}$ and can be written as $\gamma_{\mathrm{n}, \mathrm{t}}=2 \alpha_{\mathrm{n}, \mathrm{t}} \sqrt{m k_{\mathrm{n}, \mathrm{t}}}$, where $\alpha_{n, t}$ represent the dissipation coefficients defined by $\alpha_{\mathrm{n}, \mathrm{t}}=-\ln \left(e_{\mathrm{n}, \mathrm{t}}\right) /\left(\pi^{2}+\ln ^{2}\left(e_{\mathrm{n}, \mathrm{t}}\right)\right)^{1 / 2}$ as derived by Da Cruz [13]. In our case, the viscous dissipative damping is assumed to remain proportional to $\sqrt{m k_{\mathrm{n}, \mathrm{t}}}$, and the proportionality constants $\alpha_{n, t}$ are introduced as input parameters.

Roux and Chevoir [19] and Elata and Berryman [20] showed that in case of Hertz-Mindlin contacts, some loading paths can generate fictive energy in the system. In order to address this issue, when the normal effort $F_{\mathrm{n}}$ decreases by $\Delta F_{\mathrm{n}}$, the value of the tangential force when the normal force was $F_{\mathrm{n}}-\Delta F_{\mathrm{n}}$ is assigned to $F_{\mathrm{t}}$. These contact actions are embedded in each iteration, in order to update the correction terms of the predictor-corrector algorithm.

\subsection{Granular sample preparation}

The system parameters used for simulations are summarized in Table 1. It is worthwhile noticing that the surface interaction between the granular particles and the wall are modeled using the same contact equations presented in Section 2.1, where the wall is assumed to be made of steel with a Young modulus, $E=210 \mathrm{GPa}$ and a Poisson's ratio, $v=0.3$. The granular particles are made of diorite ${ }^{1}$. Unlike Alanso-Marroquin and Hermann [21] and Sitharam [22] who used reduced rigidities which produce time steps from $1^{-3} \mathrm{~s}$ to $1^{-5} \mathrm{~s}$, the simulations conducted in this study use the material properties, contact parameters and dimensions

\footnotetext{
${ }^{1}$ A grey to dark rock composed principally of plagioclase feldspar, hornblende, and/or pyroxene. Because of its hardness, diorite is widely used in road pavements and railway track platforms.
} 
Table 1

System parameters and material properties used for simulation

\begin{tabular}{|c|c|c|c|c|c|}
\hline \multicolumn{3}{|l|}{ Dimensions } & \multicolumn{3}{|l|}{ Properties } \\
\hline Initial relative density & $\phi_{\mathrm{i}}$ & 0.5615 & Particle density & $\rho_{\mathrm{p}}$ & $2710 \mathrm{~kg} / \mathrm{m}^{3}$ \\
\hline Mean particle radius & $\bar{r}$ & $3.2 \mathrm{~mm}$ & Particle Young modulus & $E$ & $46.9 \mathrm{GPa}$ \\
\hline Radius standard deviation & $\sigma_{\mathrm{r}}$ & $5.6510^{-4}$ & Particle Poisson's ratio & $v$ & 0.25 \\
\hline Radius of the cylindrical container & $R$ & $75 \mathrm{~mm}$ & Friction coefficient & $\mu$ & 0.5 \\
\hline Depth of the sample & $H$ & $60 \mathrm{~mm}$ & viscous coefficients & $\alpha_{n}, \alpha_{t}$ & 0.44 \\
\hline
\end{tabular}

presented in Table 1. This means that the step time is of about $10^{-7} \mathrm{~s}$. This step time is of the same order as the one used by Lobo-Gerrero et al. [23], it ensures realistic deformation and granular flow with respect to time (Fig. 1).

Although the particles are randomly distributed in terms of positions and shapes, the cylindrical sample is assumed to be symmetric with respect to $x$ and $y$ axes, as can be seen in Fig. 2. Therefore, only one quarter of the sample is modeled. The above mentioned figure also shows the boundary conditions concerning the planes of symmetry $\left(u_{x}=0\right.$ at $x=0$ and $u_{y}=0$ at $y=0$ ) and the wall frontier (wall-particles interactions will be described later). The cyclic load is described with a sinusoidal law as can be seen in the above mentioned Fig. 2.

We first start with the description of the sample preparation. The preparation process consists in starting with an initial configuration where the particles are randomly spread in the container with an initial density $\phi_{\mathrm{i}}$ of about 0.05 . The particles are then subjected to the gravity field in order to settle them down until the full equilibrium. In order to avoid any instability due to the preparation process, an equilibrium criterion in terms of the potential $\left(E_{\mathrm{p}}(t)\right)$ and kinetic $\left(E_{\mathrm{c}}(t)\right)$ energies is adopted, $\frac{\left\|E_{\mathrm{c}}(t)\right\|}{\left\|E_{\mathrm{p}}(t)\right\|} \leqslant \epsilon$, where $\epsilon$ is a small constant of about $10^{-6}$. At the end of the preparation process, the ratio defined beforehand, the coordination number, the apparent weight and the density of the sample are stable (Fig. 3).

As preliminary verifications, it can be noticed in Fig. 3a that the apparent weight is close to $13.6 \mathrm{~N}$, the cumulative weight of the granular sample. The small difference is due to the reaction forces at the wall-sample interface [24]. On the other hand, Fig. 3 b shows the variation of the coordination number versus time, and it obviously increases
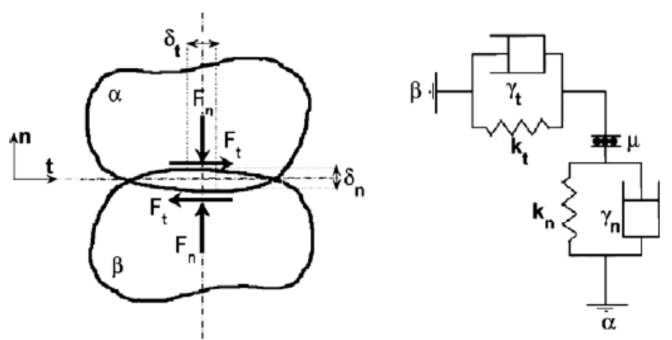

Fig. 1. Contact model for deformable bodies. with respect to time. In addition, it can be seen that the coordination number approaches 5 , when the sample stabilizes. Actually, in order to obtain a stable sample, each particle should have a sufficient number of constraints. According to Alexander [25], in a frictionless assembly, the coordination number in a $\mathscr{D}$ dimensional space should be at least equal to $z=2 \mathscr{D}$. However, in a frictional assembly, Edwards [26] asserted that this number is at least equal to $z=1+\mathscr{D}$. The samples prepared herein are subjected to a friction coefficient of 0.5 , therefore the obtained results are in agreement with the above mentioned propositions. According to Suiker and Fleck [27], the coordination number of 3D granular systems depends on the friction coefficient. In addition, it falls between 4 and 6 when the particles are relatively rigid, monodisperse, rotating, and in neutral equilibrium. This behavior is mainly due to the frictional energy dissipation which increases with $\mu$ and induces a slowing down of the rearrangement process. In Fig. 3c the granular relative density is evaluated with respect to time. It can be noticed that the relative density stabilizes at the end of the preparation process and asymptotically tends to $\phi_{f}=0.53^{2}$. The former value justifies the use of the material properties presented in Table 1. Actually, the authors noticed that reducing the material properties results in soft contact stiffness and induces high particle penetrations. This leads to densities which are too high with no physical meaning. As a matter of fact, a randomly distributed granular package can be stable under loading starting from a compaction relative density $\phi_{\mathrm{lp}}$ of $0.555 \pm 0.005$ (loose packing [28]) until $\phi_{\mathrm{dp}}=0.64$ (dense packing). Crystalline solids with consistent three-dimensional orders in their internal structure can reach a maximum relative density of $\phi_{\mathrm{cl}}=0.74$. The former value seems to be the limit that cannot be overstepped for real materials. In practice, densities from $\phi_{\mathrm{dp}}$ and $\phi_{\mathrm{cl}}$ are difficult to reach unless artificially arranged in order to get an ordered structure.

\section{Residual displacement estimation}

When used during loading cycles, the molecular dynamics method briefly described in the last section provides enough information to estimate the granular configuration

\footnotetext{
${ }^{2}$ The free surface of the sample is non uniform after preparation, this induces an overestimated sample height and consequently gives an underestimated relative density. The more accurate relative density is about $\phi=0.5615$.
} 
a

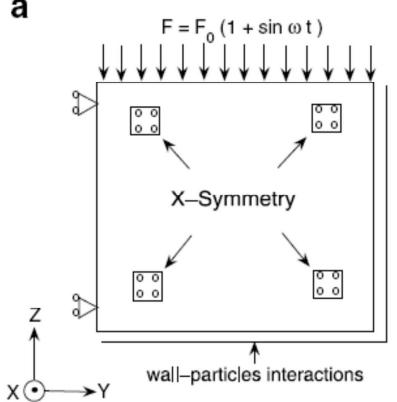

b A quarter of a granular sample prepared using

the molecular dynamics under the gravity field

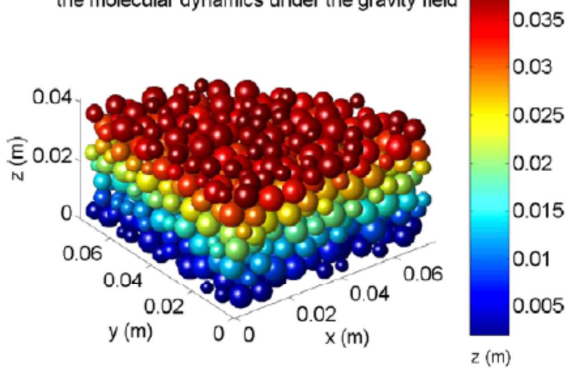

Fig. 2. Loads and boundary conditions (a) applied to the granular material sample (b).
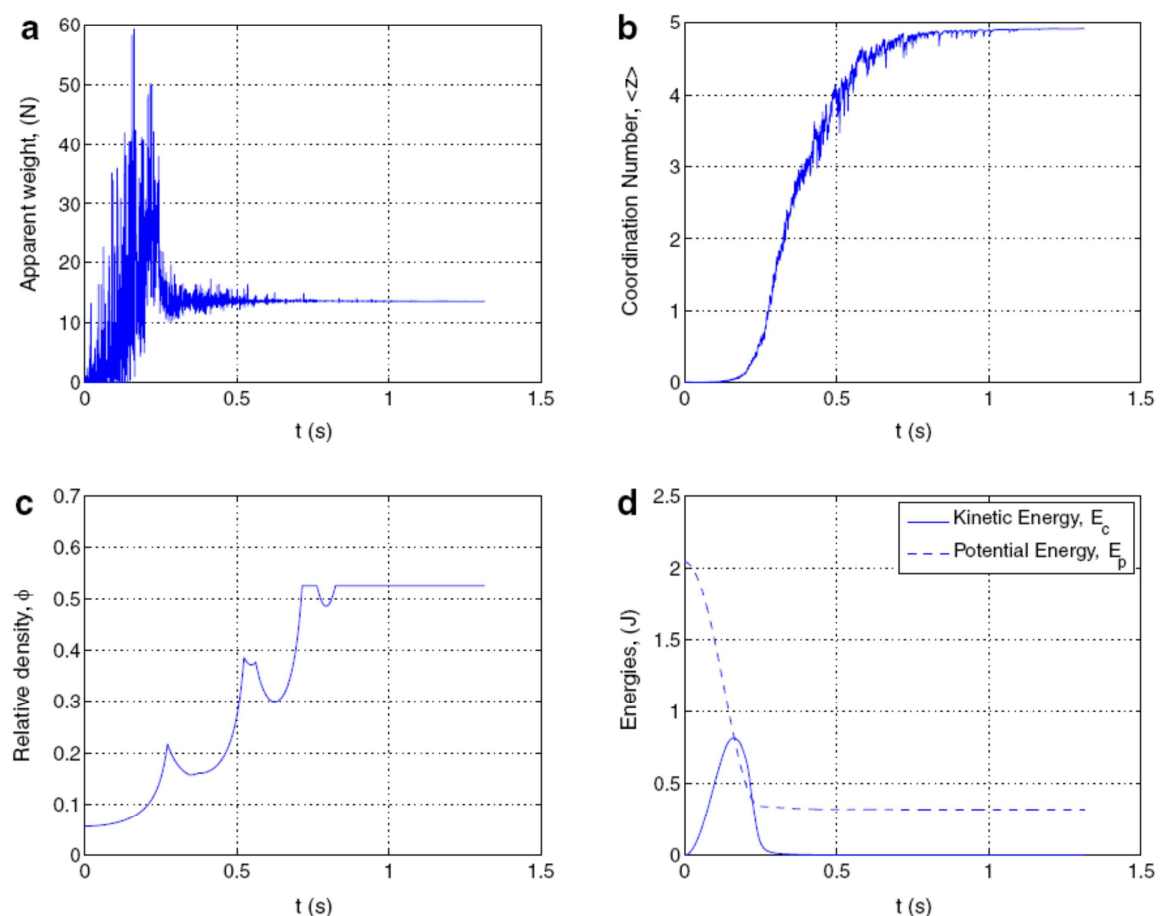

Fig. 3. Sample preparation under gravity field variation of: (a) apparent weight, (b) coordinations number, (c) granular relative density, and (d) kinetic energy (resp. potential energy) versus time.

after a finite number of cycles. In this section, we explain the time averaging technique and we compare its results to a full time integration for several numbers of cycles.

\subsection{Estimation method}

Let us subject the sample $\mathscr{S}$ to a cyclic load of period $T$, then the particles flow according to the initial chain of contacts, internal interactions, material properties, and boundary conditions. The displacements of the particles with respect to time can be additively decomposed as follows: $\left\{\begin{array}{l}\forall t \in[0, T], \quad \forall \beta \in \mathscr{S}, \\ u_{\beta}(t)=u_{\beta}^{\mathrm{r}}(t)+u_{\beta}^{\mathrm{v}}(t),\end{array}\right.$

where the superscripts (r) and (v) denote, respectively, the residual and reversible displacements. It should be emphasized that the loading cycle results in dynamic and viscoelastic effects that continue to act even after removing the exciting load. This means that they may continue to move and to explore new positions until a final equilibrium is reached. Once the dynamic and viscoelastic effects vanish, the residual displacement of the particle $\beta$ can be simply 

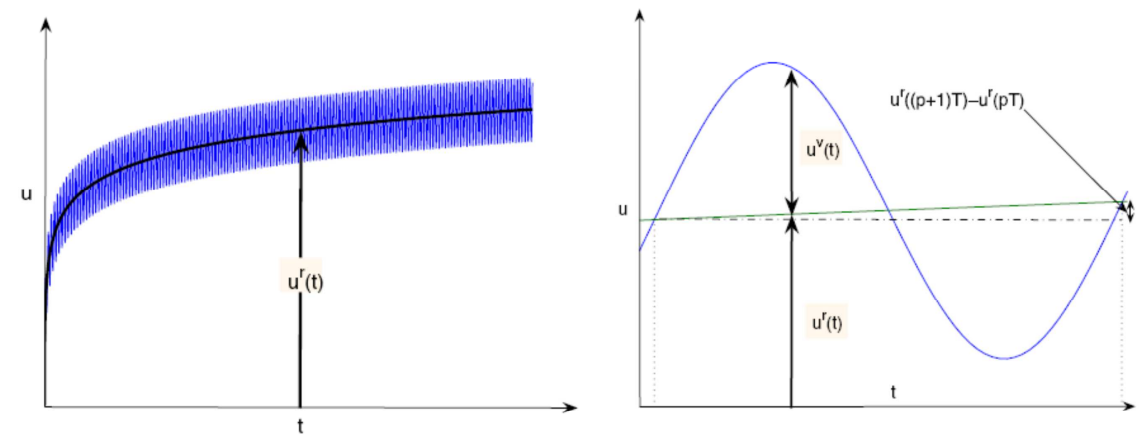

Fig. 4. Overall displacement evolution with respect to time, illustration of the residual and reversible displacement.

described as the difference between the initial and final (obtained after cyclic loading and relaxation) displacements:

$u_{\beta}^{\mathrm{r}}(T)=\lim _{\tau \rightarrow+\infty}\left(u_{\beta}(T+\tau)-u_{\beta}(0)\right)$,

where $\tau$ represents the relaxation time. It can be easily noticed that anticipating the residual displacement at the end of the loading cycle is not an obvious task. On the other hand, the above mentioned definition is not suitable for the calculation of instantaneous residual displacements, especially when the sample is subjected to several loading cycles with no relaxing time in between.

In order to overcome this problem and suggest an appropriate residual displacement description, let us investigate a typical overall displacement of granular samples subjected to repeated loading. According to observations made on granular materials, the overall displacement can be illustrated by a typical description presented in Fig. 4. In this representation, the first term of Eq. (8) represents the residual displacement which increases slowly with time and the second denotes the fluctuating reversible displacement with respect to time.

It can be seen that the differential residual displacement over a cycle $p$ is considerably smaller than the reversible displacement as argued by Abdelkrim [6]:

$$
\begin{aligned}
& \left\|u_{\beta}^{\mathrm{r}}((p+1) T)-u_{\beta}^{\mathrm{r}}(p T)\right\| \ll \max \left(\left\|u_{\beta}^{\mathrm{e}}(t)\right\|\right), \\
& \quad t \in[p T,(p+1) T] .
\end{aligned}
$$

Therefore, the overall displacement can be seen as a rapidly fluctuating periodic function representing the reversible term, a slowly increasing function representing the residual term, and a correcting term representing the error that can be encountered. It is worthwhile to notice that the error term represents the unpredicted or not explained variation in the dependent variable. It is conventionally called "error" whether it is really measurable or not. Since the reversible term is periodic, the displacement can be deduced from Eq. (8) as follows:

$u_{\beta}(t+T)=u_{\beta}(t)+u_{\beta}^{\mathrm{r}}(t+T)+\xi_{\beta}$.
Over several loading cycles, $p=1, \ldots, k$, the displacement of the particle $\beta$ under consideration can be written as follows:

$$
\begin{aligned}
u_{\beta}(t+k T)= & u_{\beta}(t)+\sum_{p=1}^{k}\left[u_{\beta}^{\mathrm{r}}(t+p T)-u_{\beta}^{\mathrm{r}}(t+(p-1) T)\right. \\
& \left.+\xi_{\beta}^{(p)}-\xi_{\beta}^{(p-1)}\right] .
\end{aligned}
$$

Now, the objective is to estimate the unknown function $u_{\beta}^{\mathrm{r}}(t)$, where $\xi_{\beta}$ is a zero mean random error. The estimation is made based on a finite number of training data ${ }^{3}$ $u(t+T), \ldots, u(t+k T)$. It provides the best functions $\tilde{u}_{\beta}^{\mathrm{r}}(t)$ expressed in terms of a set of parameters $a$ which can be obtained by the minimum square root method.

Several functions can be chosen to describe and estimate the residual displacement $u_{\beta}^{\mathrm{r}}(t)$. For instance, this function can be assumed to be linear with respect to time, in order to simplify the presentation without loss of generality. Then, the accuracy of the estimation can be tested by studying the variation of the following functions with respect to the number of cycles:

$g_{u}(k)=\frac{1}{k}\left(\sum_{\beta, p, d}\left[u_{\beta, d}^{\mathrm{r}}(t+p T)-u_{\beta, d}^{\mathrm{r}}(t+(p-1) T)\right]^{2}\right)^{\frac{1}{2}}$

and

$f_{\xi}(k)=\frac{1}{k}\left(\sum_{\beta, p, d}\left[\xi_{\beta, d}^{p}\right]^{2}\right)^{\frac{1}{2}}$,

where $\beta$ denotes the particles of the systems, $p$ represents the index of a given training datum, and $d$ one of the three degree of translation. The two above mentioned functions are simply the expressions of the second norm used to study the accuracy of the estimations. In Fig. 5, it can be

\footnotetext{
${ }^{3}$ The term "training data" is widely encountered in neural network applications. It will be used herein to denote the displacements of the particles with respect to the number of cycles. These displacements are called training data since they will be used first for estimation and then for extrapolation
} 

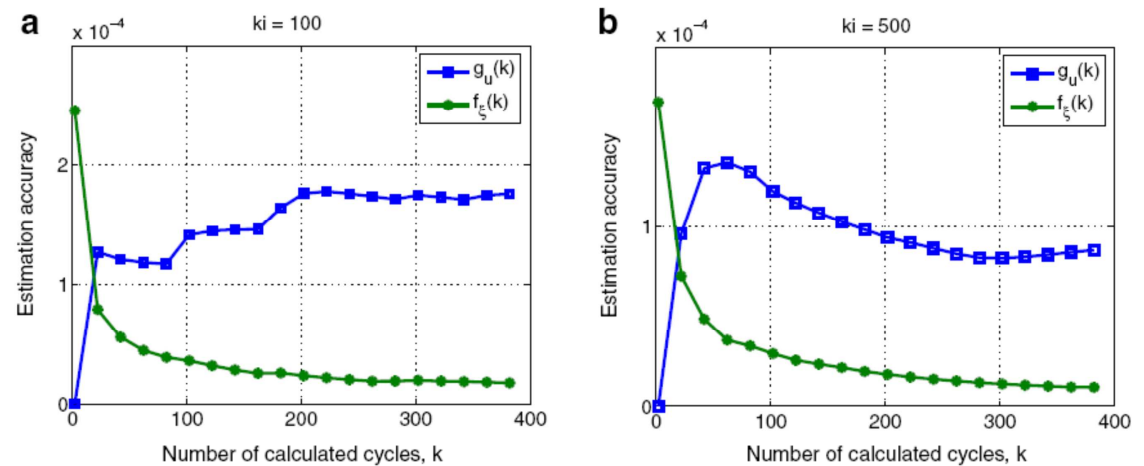

Fig. 5. Accuracy of the estimation procedure for a linear estimator with two different initial numbers of calculated cycles, $k_{\mathrm{i}}$.

seen that $g_{u}$ becomes stable when $k$ increases, moreover, the error terms decrease in norm average $f_{\xi}$ when $k$ increases.

The number of training data required for the estimation of the trend function can be expressed in such a way that the accuracy is controlled. In general, applying the law of large numbers provides an upper bound which converges in $\frac{1}{\sqrt{n}}$. In our case, a higher bound can be obtained since the errors are always bounded (the errors are within the maximum and minimum displacements obtained along $k$ loading cycles). The sequence of error terms $\left(\xi_{\beta}^{(p)}\right)_{p=1, \ldots, k}$ can be seen as a set of random independent variables, where $\xi_{\beta}^{(p)} \in[a, b]$. The probability of obtaining a function which estimates the residual displacement with an error $\epsilon$ can be written as

$\operatorname{Pr}\left[\left\|\frac{1}{k} \sum_{p} \xi_{\beta}^{(p)}\right\| \geqslant \epsilon\right] \leqslant 2 \mathrm{e}^{-\frac{2 k \epsilon^{2}}{(b-a)^{2}}}$.

The above mentioned inequality is a theorem which is known as the "Chernoff Bound". Its proof can be found in [29]. According to Eq. (15), it is possible to control the necessary number of cycles, $k$, by choosing an error level of $\epsilon$ and an associated probability. Indeed, for $k \geqslant \frac{(b-a)^{2}}{2 \epsilon^{2}} \ln \left(\frac{2}{p}\right), \operatorname{Pr}\left[\left\|\frac{1}{k} \sum_{p} \xi_{\beta}^{(p)}\right\| \geqslant \epsilon\right] \leqslant p$. The trend function can be estimated using the available training data within the preselected error.

So far, the accuracy of the estimations is established through a parametric approach. The obtained model estimating the residual displacements over $k$ loading cycles will be used in the following section in order to extrapolate the results and obtain an estimated configuration after additional $h$ loading cycles.

\subsection{Extrapolation}

Using the estimation model obtained beforehand, the final configuration as well as the residual displacements evaluated during the above mentioned $k$ cycles, it is possi- ble to estimate the $(k+h)$ th configuration, where $h \in \mathbb{N}$. In particular, if the residual displacement is assumed to be linear with respect to time, this configuration can be described as follows:

$$
\begin{aligned}
\hat{u}_{\beta}(t+(k+h) T)= & u_{\beta}(t+k T)+\frac{h}{k} \sum_{p=1}^{k}\left[u_{\beta}^{\mathrm{r}}(t+p T)\right. \\
& \left.-u_{\beta}^{r}(t+(p-1) T)\right] .
\end{aligned}
$$

The first right-hand term of the above mentioned equation is known from the last step, whereas, the second represents the estimation of the residual displacement at the $(k+h)$ th cycle. The estimation suggested beforehand assumes that the residual displacement is linear with respect to the number of cycles. A better estimation can be made using a more appropriate settlement law that has been obtained empirically by Shenton [1]. This law reads $\tilde{u}_{\beta}^{r}(t+p T)=a_{\beta} \ln$ $(t+p T)+b_{\beta}(t+p T)$. The parameters $a_{\beta}$ and $b_{\beta}$ can be deduced from the molecular dynamics calculations using the least square method (minimizing the quadratic error between the empirical low and the calculated residual displacements). Therefore, the displacement can be written as follows:

$$
\begin{aligned}
\hat{u}_{\beta}(t+(k+h) T)= & u_{\beta}(t+k T)+a_{\beta} \ln (t+(k+h) T) \\
& +b_{\beta}(t+(k+h) T),
\end{aligned}
$$

where the parameters $a_{\beta}$ and $b_{\beta}$ are calculated using the least squares method which consists in minimizing the squares of errors expressed by:

$\mathscr{R}=\sum_{p=1}^{k}\left(\xi_{\beta}^{p}\right)^{2}=\sum_{p=1}^{k}\left(u_{\beta}^{\mathrm{r}}(t+p T)-\tilde{u}_{\beta}^{\mathrm{r}}(t+p T)\right)^{2}$.

The above mentioned expression is a second-order polynom, its minimum can be obtained by deriving it with respect to its variables $a_{\beta}$ and $b_{\beta}$ : 


$$
\begin{aligned}
\frac{\partial R}{\partial a_{\beta}}= & -2 \sum_{p=1}^{k}\left[u_{\beta}^{\mathrm{r}}(t+p T)-a_{\beta} \ln (t+p T)\right. \\
& \left.-b_{\beta}(t+p T)\right] \operatorname{Ln}(t+p T)=0, \\
\frac{\partial R}{\partial b_{\beta}}= & -2 \sum_{p=1}^{k}\left[u_{\beta}^{\mathrm{r}}(t+p T)-a_{\beta} \ln (t+p T)\right. \\
& \left.-b_{\beta}(t+p T)\right](t+p T)=0 .
\end{aligned}
$$

This system of two unknowns and two equations can be solved to obtain the following expression of the estimation parameters, using the information collected during the molecular dynamics calculations:

$a_{\beta}=\frac{\sum_{p=1}^{p=k} t_{p}^{2} \sum_{p=1}^{p=k} u_{\beta}^{\mathrm{r}}\left(t_{p}\right) \ln \left(t_{p}\right)-\sum_{p=1}^{p=k} t_{p} \ln \left(t_{p}\right) \sum_{p=1}^{p=k} u_{\beta}^{r}\left(t_{p}\right) t_{p}}{\sum_{p=1}^{p=k} \ln ^{2}\left(t_{p}\right) \sum_{p=1}^{p=k} t_{p}^{2}-\left(\sum_{p=1}^{p=k} t_{p} \ln \left(t_{p}\right)\right)^{2}}$

and

$b_{\beta}=\frac{\sum_{p=1}^{p=k} \ln \left(t_{p}\right)^{2} \sum_{p=1}^{p=k} u_{\beta}^{r}\left(t_{p}\right) t_{p}-\sum_{p=1}^{p=k} t_{p} \ln \left(t_{p}\right) \sum_{p=1}^{p=k} u_{\beta}^{r}\left(t_{p}\right) \ln \left(t_{p}\right)}{\sum_{p=1}^{p=k} \ln ^{2}\left(t_{p}\right) \sum_{p=1}^{p=k} t_{p}^{2}-\left(\sum_{p=1}^{p=k} t_{p} \ln \left(t_{p}\right)\right)^{2}}$,

where $t_{p}=t+p T$. To distinguish between the two approaches, Eq. (16) will be termed as linear estimation and Eq. (17) will be called logarithmic estimation. It is obvious that for both of the above mentioned approaches, the closer $h / k$ is to zero, the better the estimated configuration is. However, the objective of the procedure is to simulate the granular flow when $h$ is close to or higher than $k$, therefore, the parameters will be selected in such a way that the estimation procedure gives sufficiently accurate results.

\subsection{Numerical application}

In order to examine the reliability of the time averaging technique, estimated configurations are compared to full time calculated solutions. The prepared stable sample is now subjected successively to $k_{i}$ (initial) and $k$ loading cycles, as described beforehand. The information collected from the molecular dynamics calculation is used in order to estimate the configuration after $h$ cycles as described in the last section. In order to systematically evaluate the agreement between the calculated and estimated displacements, the following estimation of the relative error $\mathscr{E}_{r}$ is defined:

$$
\mathscr{E}_{r}(u)=\frac{\|u-\hat{u}\|}{\|u\|}
$$

The second norm, as defined in Eqs. (13) and (14), is used in the above mentioned equation in order to calculate the relative error. Note that $u$ is an $N \times 3$ matrix containing the coordinates of the particles. It denotes the calculated residual displacements. Similarly, $\hat{u}$ represents the estimated residual displacements, $\beta$ is a particle of the system
$\mathscr{S}$, and $N$ is the number of particles. Figs. 6 and 7 show that an interesting agreement between the calculated and estimated configurations is obtained, especially when the calculated number of cycles $k$ is higher than 20, either for the linear or logarithmic estimation approaches. It can be seen in the above mentioned figures that the error in these cases does not exceed 2\% (in Figs. 6 and 7, the errors are presented in a logarithmic scale for clarity).

It can also be noticed that the initial number of cycles $k_{\mathrm{i}}$ highly affects the accuracy of the estimation techniques. Fig. 6 shows that the maximum relative error decreases from $14 \%$ to $2.5 \%$ when $k_{\mathrm{i}}$ increases from 10 to 500 initial loading cycles, in the case of linear estimation. Similarly, Fig. 7 shows that the maximum relative error decreases from $14 \%$ to $1 \%$ when $k_{\mathrm{i}}$ increases from 10 to 500 initial loading cycles, in the case of a logarithmic estimation. In addition, Figs. 6 and 7 show that the relative estimation errors decrease with the number of calculated cycles $k$. This is obviously due to the fact that the more calculated points are available, the better are the estimations. Moreover, it can be seen that the relative error increases with the length of the estimation interval $h$. More interestingly, it can be noticed that the logarithmic estimation is more accurate than the linear estimation. Actually, with its two parameters, this approach is more appropriate for time averaging. Therefore, it will be used in the last section in the full settlement calculation procedure.

In spite of the accurate residual displacements that can be obtained through the averaging technique, the obtained configuration can exhibit local excessive penetrations between the particles, especially for large numbers of cycles $h$. This kind of configuration may be unsuitable for further molecular dynamics calculations. In order to overcome this problem, a relaxation technique which consists of correcting the estimated configuration is suggested in the following section.

\section{Relaxation method}

The objective of this section is to prove the existence of an optimality property that can be satisfied by the boundary conditions of the system $\mathscr{S}$ as well as the equations of motions (1). This property is used in order to equilibrate instable configurations with excessive local penetrations.

\subsection{Minimum energy functional}

Applying an arbitrary vector $u_{\beta} \in \mathbb{R}^{3}$ to the equations of motion (1), it is possible to establish its dual, the virtual work principle (v.w.p.) [30], which can be written as follows:

$$
\left\{\begin{array}{l}
\forall\left(u_{\beta}\right)_{\beta \in \mathscr{S}} \in\left(\mathbb{R}^{3}\right)^{N}, \\
\sum_{\beta \in \mathscr{S}} F_{\beta} u_{\beta}+\sum_{\beta \in \mathscr{S}} \sum_{\alpha \neq \beta \in \mathscr{S}} F_{\alpha \beta} u_{\beta}=\sum_{\beta \in \mathscr{S}} m_{\beta} a_{\beta} u_{\beta} .
\end{array}\right.
$$

The first left-hand term of the above mentioned equation is the virtual work of the external forces, it can be expressed by $\mathscr{P}_{(\mathrm{e})}\left(u_{1}, \ldots, u_{N}\right)=\sum_{\beta \in \mathscr{S}} F_{\beta} u_{\beta}$. The second 

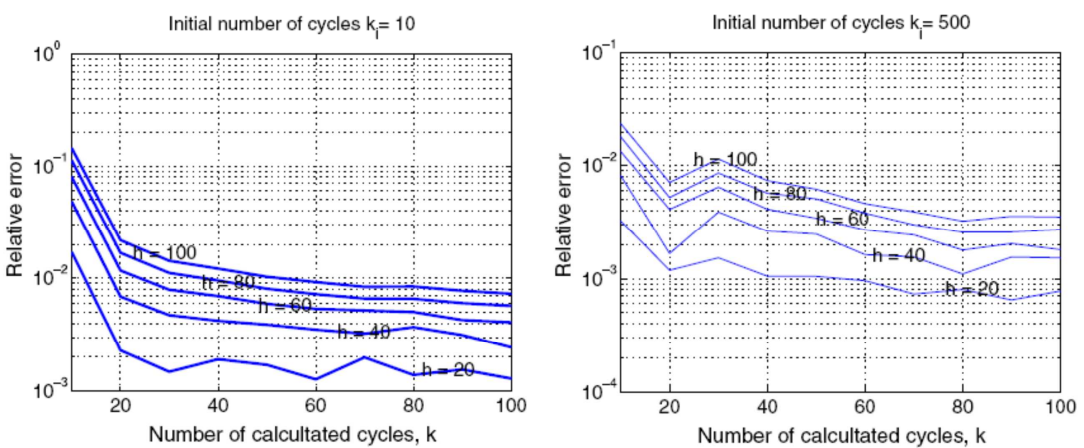

Fig. 6. Linear estimation: relative error distributions for different initial number of cycles $\left(k_{\mathrm{i}}\right)$, calculated number of cycles $(k)$, and extrapolated number of cycles $(h)$.
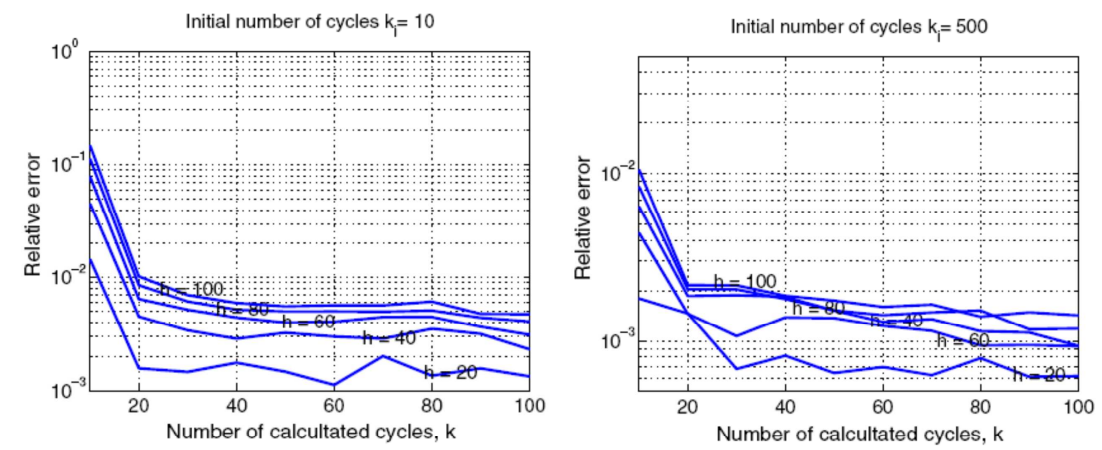

Fig. 7. Logarithmic estimation: relative error distributions for different initial number of cycles $\left(k_{\mathrm{i}}\right)$, calculated number of cycles $(k)$, and extrapolated number of cycles $(h)$.

left-hand term expresses the virtual work of the internal forces $\mathscr{P}_{(\mathrm{i})}\left(u_{1}, \ldots, u_{N}\right)=\sum_{\beta \in \mathscr{G}} \sum_{\alpha \neq \beta \in \mathscr{S}} F_{\alpha \beta} u_{\beta}$. The right-hand term denotes the work of the inertia forces $\mathscr{A}\left(u_{1}, \ldots, u_{N}\right)=\sum_{\beta \in \mathscr{S}} m_{\beta} a_{\beta} u_{\beta}$. Note that the set of vectors $\left(u_{\beta}\right)_{\beta \in[1, N]]}$ is arbitrary in $\left(\mathbb{R}^{3}\right)^{N}$. Therefore, it can be seen as virtual displacements or velocity applied to the system $\mathscr{S}$. In particular, this virtual displacement can be taken continuous and in coherence with the boundary conditions $\left(u_{\beta}=u_{\beta}^{\mathrm{d}} \forall \beta \in \overline{\mathscr{S}}^{\mathrm{d}}\right)$, where $\overline{\mathscr{S}^{\mathrm{d}}}$ denotes the frontier of $\mathscr{S}$ where displacements are applied. Similarly, the external forces of Eq. (1) should be in coherence with the boundary conditions $\left(F_{\beta}=F_{\beta}^{\mathrm{f}} \forall \beta \in \overline{\mathscr{S}^{\mathrm{f}}}\right)$, where $\overline{\mathscr{S}^{\mathrm{f}}}$ denotes the frontier of $\mathscr{S}$ where forces are applied.

Applying a set of displacements $\left(u_{\beta}\right)_{\beta \in[[1, N]]}$ to the system of particles $\mathscr{S}$, the existence of a solution for the equations of motion implies that there is a set of internal forces $\left(F_{\alpha \beta}\right)_{\beta, \alpha \in\|1, N\|^{2}}$ and a set of external forces $\left(F_{\beta}\right)_{\beta \in[1, N]]}$ governing the evolution problem. Taking into consideration the above mentioned solution $\left(u_{\beta}, F_{\beta}, F_{\alpha \beta}\right)_{\beta, \alpha \in\left[1, N \|^{2}\right.}$, it is possible to write the v.w.p. as follows:

$$
\left\{\begin{array}{l}
\sum_{\beta \in \overline{\mathscr{F}}} F_{\beta}^{\mathrm{f}} u_{\beta}+\sum_{\beta \in \overline{\mathscr{S}}} F_{\beta} u_{\beta}^{\mathrm{d}}+\sum_{\beta \in \mathscr{T}} m_{\beta}(g-a) u_{\beta}, \\
+\sum_{\beta \in \mathscr{S}} \sum_{\alpha \neq \beta \in \mathscr{S}} F_{\alpha \beta} u_{\beta}=0 .
\end{array}\right.
$$

where $\overline{\mathscr{S}} \mathrm{d} \overline{\mathscr{S}}^{\mathrm{r}}=\mathscr{S}$ and $g$ represents the gravity acceleration. As a second application of the v.w.p., let us consider the same set of forces, the solution of Eq. (1) associated with a different virtual displacement, which is in coherence with the boundary conditions, $\left(u_{\beta}^{\prime}, F_{\beta}, F_{\alpha \beta}\right)_{\beta, \alpha \in\|1, N\|^{2}}$. This leads to a second equation which is similar to Eq. (24). The difference between them leads to the following equation:

$$
\left\{\begin{array}{l}
\sum_{\beta \in \overline{\mathscr{F}}}\left(F_{\beta}^{\mathrm{f}} u_{\beta}^{\prime}-F_{\beta}^{\mathrm{f}} u_{\beta}\right)+\sum_{\beta \in \mathscr{T}} m_{\beta}(g-a)\left(u_{\beta}^{\prime}-u_{\beta}\right), \\
+\sum_{\beta \in \mathscr{S}} \sum_{\alpha \neq \beta \in \mathscr{S}}\left(F_{\alpha \beta} u_{\beta}^{\prime}-F_{\alpha \beta} u_{\beta}\right)=0 .
\end{array}\right.
$$

The last left-hand term of Eq. (25) can be expressed as follows:

$$
\left\{\begin{array}{l}
\forall \alpha, \beta \in \mathscr{T}, \quad \text { in contact, } \\
F_{\alpha \beta}\left(u_{\alpha}, u_{\beta}\right)=\nabla \psi_{\alpha \beta}\left(u_{\alpha}, u_{\beta}\right),
\end{array}\right.
$$

where $\psi_{\alpha \beta}\left(u_{\alpha}, u_{\beta}\right)$ represents the potential of the contact forces. The proof of the optimum existence can be established through the variation rate of this potential:

$\delta \psi_{\alpha \beta}\left(u_{\alpha}, u_{\beta}\right)=\psi_{\alpha \beta}\left(u_{\alpha}+\delta u_{\alpha}, u_{\beta}+\delta u_{\beta}\right)-\psi_{\alpha \beta}\left(u_{\alpha}, u_{\beta}\right)$. 
Considering a quasi-static relaxation, the condition $\left\|F_{\mathrm{t}}\right\| \leqslant \mu F_{\mathrm{n}}$ remains valid and it is possible to write the potential as $\psi_{\alpha \beta}\left(u_{\alpha}, u_{\beta}\right)=\frac{k_{n}}{\gamma+1} \delta_{\mathrm{n}}^{\gamma+1}\left(u_{\alpha}, u_{\beta}\right)$, where $\gamma$ depends on the normal contact model, it equals $3 / 2$ in case of Hertz law, $\delta_{\mathrm{n}}\left(u_{\alpha}, u_{\beta}\right)=\left[0.5\left(d_{\alpha}+d_{\alpha}\right)-\left\|x_{\alpha}+u_{\alpha}-x_{\beta}+u_{\beta}\right\|\right]$, and $x_{\beta \in[[1, N]]}$ are the initial positions of the particles. Making use of Cauchy-Schwartz inequality, it is possible to show that $\delta \psi_{\alpha \beta} \geqslant 0$ while contact exists. Therefore, it can be deduced that:

$$
\left\{\begin{array}{l}
\forall \text { Solution } \quad\left(u_{\beta}, F_{\beta}, F_{\alpha \beta}\right)_{\beta, \alpha \in \| 1, N \rrbracket^{2}}, \\
\forall u^{\prime} \neq u \text { in coherence with } u^{d}, \\
\sum_{\beta \in \overline{\mathscr{T}}} F_{\beta}^{\mathrm{f}} u_{\beta}^{\prime}+\sum_{\beta \in \mathscr{S}} m_{\beta} g u_{\beta}^{\prime}+\sum_{\beta \in \mathscr{S}} \sum_{\alpha \neq \beta \in \mathscr{S}} \psi_{\alpha \beta}\left(u_{\beta}^{\prime}\right), \\
\geqslant \sum_{\beta \in \overline{\mathscr{T}}} F_{\beta}^{\mathrm{f}} u_{\beta}+\sum_{\beta \in \mathscr{S}} m_{\beta} g u_{\beta}+\sum_{\beta \in \mathscr{S}} \sum_{\alpha \neq \beta \in \mathscr{S}} \psi_{\alpha \beta}\left(u_{\beta}\right) .
\end{array}\right.
$$

The obtained inequality proves the existence of a functional $\mathscr{F}(u)$ which reads

$$
\mathscr{F}(u)=\sum_{\beta \in \overline{\mathscr{T}} f} F_{\beta}^{f} u_{\beta}+\sum_{\beta \in \mathscr{T}} m_{\beta} g u_{\beta}+\sum_{\beta \in \mathscr{S}} \sum_{\alpha \neq \beta \in \mathscr{S}} \psi_{\alpha \beta}\left(u_{\beta}\right) .
$$

This functional can be minimized in order to equilibrate instable configurations where excessive penetrations in some zones can induce divergence of the flow.

\subsection{Numerical application}

In this section, we consider particular instable configurations characterized by local excessive penetrations and subject them to the relaxation technique which consists of minimizing the functional $\mathscr{F}(u)$ while keeping fixed all the particles of the frontier, in order to find out the optimal particle positions guaranteeing a stable system $\mathscr{S}$. As a first example, let us consider a granular cell of nine particles in coherence with the material properties defined in Table 1 . This cell is presented in Fig. 8. The results show that this approach equilibrates the penetrations and provides a stable configuration.

Now, let us consider a second example corresponding to an estimated configuration from simulations carried out as described in the last section. The configuration under consideration is obtained after $k_{\mathrm{i}}=20$ initial cycles, $k=20$ additional calculated cycles, and a linear extrapolation over $h=20$ cycles. In terms of settlement calculations, such an estimation is in agreement with the calculated results as discussed in the last section. However, it can be seen in Fig. 9a that it exhibits excessive particle penetrations. Although limited in number, these anomalous penetrations may be important enough to induce flow divergence.

The relaxation method for this case consists of fixing all the particles of the frontier and finding the optimal positions by minimizing the functional (29) using the gradient method. It leads to an equilibrated configuration with the penetration distribution presented in Fig. 9b. It can be noticed that the distribution is improved and the maximum penetration is reduced from $1.8 \times 10^{-3} \mathrm{~m}$ to $6 \times 10^{-5} \mathrm{~m}$.

The example presented herein proves that the relaxation technique improves the estimated configuration in terms of penetration magnitudes and distributions. In the following section, the estimation and relaxation techniques will be used in a full procedure to describe the settlement of granular samples.

\section{Settlement procedure}

In this section the molecular dynamics algorithm, the averaging technique and the relaxation method, described beforehand are used in a computational procedure in order to simulate granular materials settlement under repeated loading. The flow chart of the procedure is explained, then a convergence study is conducted. Finally, a particular field case is studied in order to show the advantages and limits of the suggested procedure.

\subsection{Flow chart of the computational procedure}

The flow chart displayed in Fig. 10, transfers the basic idea of the concept into a programmable algorithm. The analysis starts with an initial configuration where the particles are randomly distributed in the cylinder with no penetration in between. This initial configuration is then
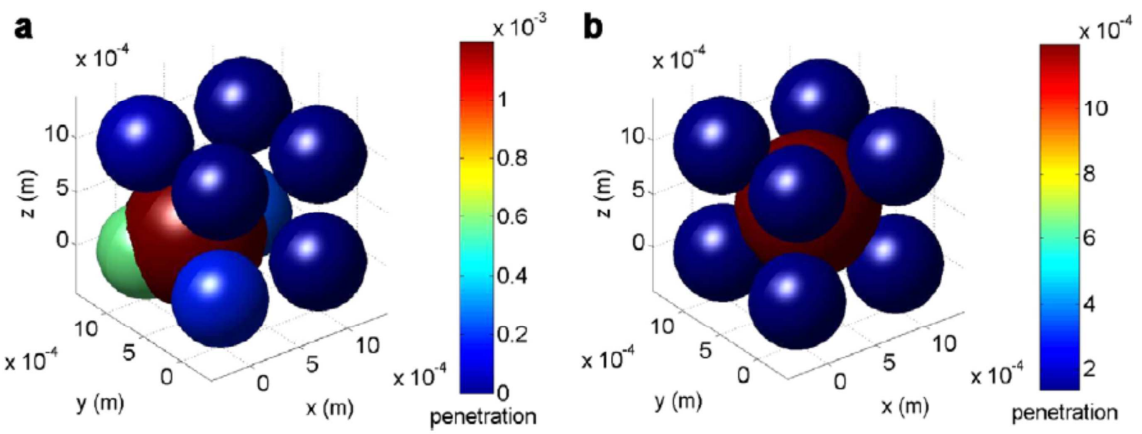

Fig. 8. Relaxation of a granular cell of nine particles: (a) estimated configuration and (b) corrected configuration. 

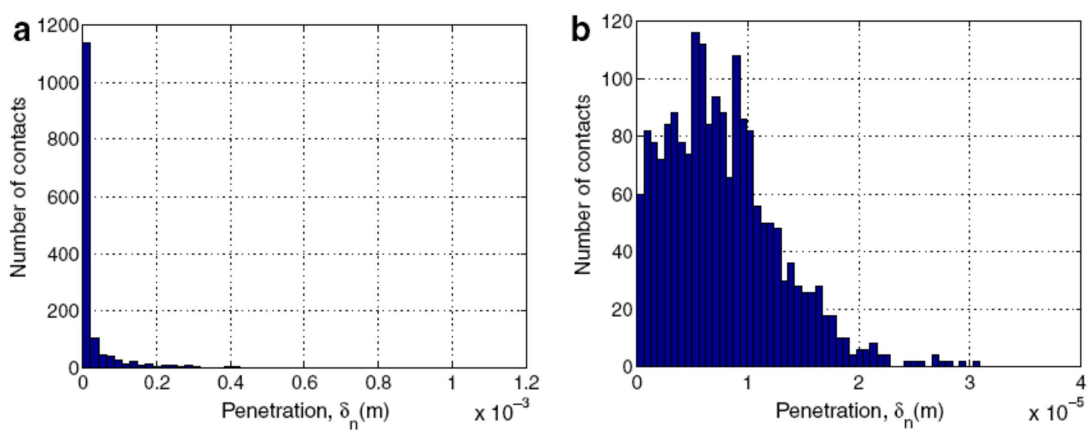

Fig. 9. Relaxation of a full sample: (a) estimated configuration and (b) corrected configuration.

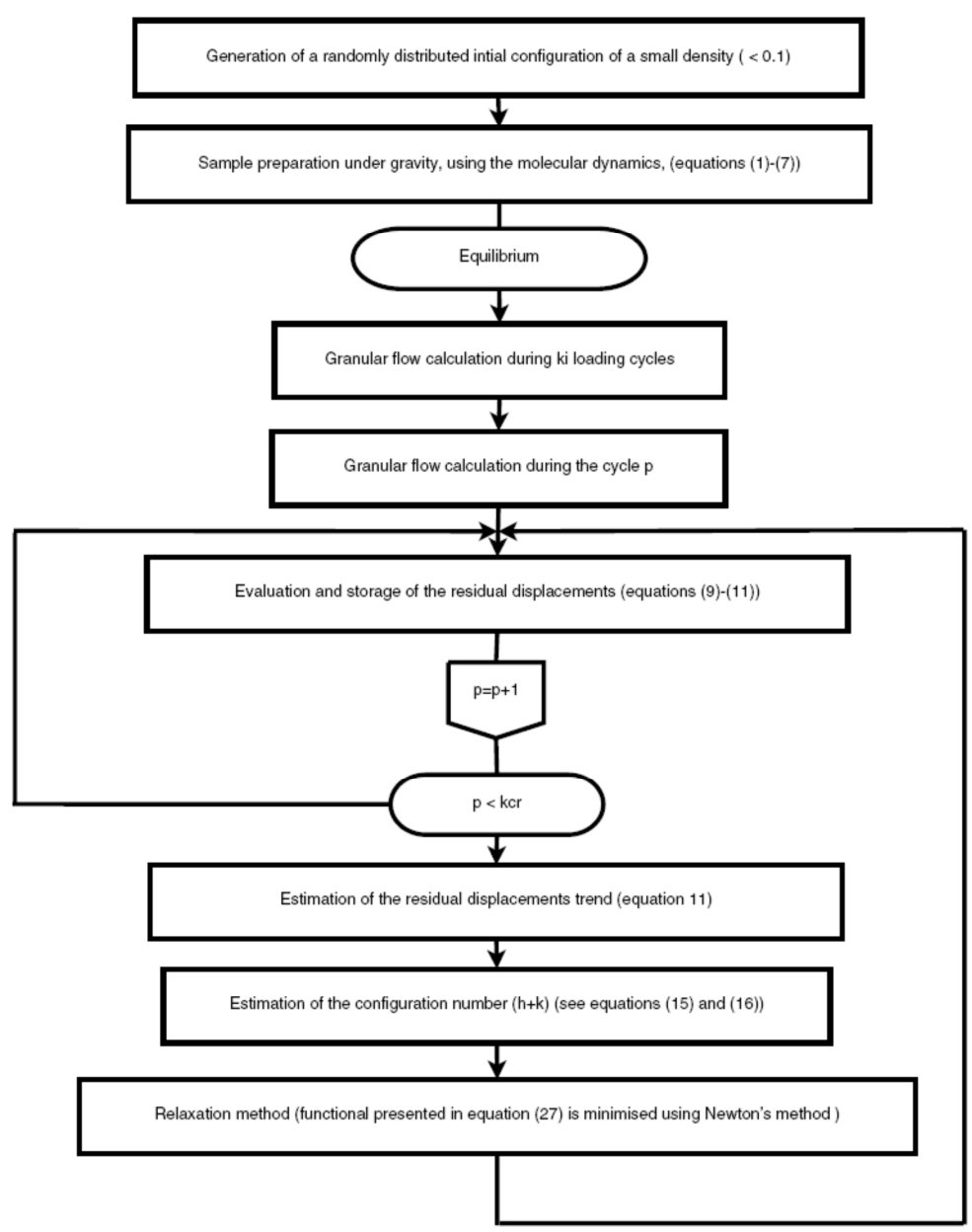

Fig. 10. Flow chart of the settlement computational procedure. 
subjected to the gravity field until equilibrium (Fig. 3). The obtained sample is subjected to $k_{\mathrm{i}}$ initial loading cycles, before starting the sequential procedure, since the first cycles are always characterized by high settlement slopes as can be seen in Fig. 4. The sequential procedure consists first in subjecting the sample to $k$ loading cycles which upper bound can be determined knowing the probability and error levels defined in Eq. (15). During these $k$ loading cycles, training data are evaluated using the molecular dynamics and stored at the end of each cycle. The collected information is then used to establish trend functions describing the behavior of the granular sample in terms of residual displacement. The obtained functions are then used for logarithmic extrapolation up to $(h+k)$ cycles, as described by Eqs. (17), (20), and (21).

Although the estimations and extrapolations are tested in terms of residual displacements accuracy, possible local anomalies in terms of excessive penetrations may show up, as can be seen in Fig. 9. Addressing these possible issues consists of applying the relaxation technique to the obtained configuration. The former step produces stable configurations which are suitable for further molecular dynamics calculations.

In Section 2, it has been shown that the accuracy of the estimation technique depends on the values of $k, k$ and $h$. The relative error decreases with the increase of $k$ and/or the decrease of $h$. In addition, the estimation accuracy increases with the total number of cycles. Actually, in terms of computational cost, it is obvious that the higher the ratio $h / k$, the better it is. It is therefore advantageous to increase $h$ with the total number of iteration. In this study, the parameter $h$ can be varied with respect to the number of iterations, since the error decreases when the total number of calculated cycles increases.

\subsection{Convergence study}

In order to validate the suggested procedure, the obtained overall residual displacements are compared to full molecular dynamics calculations. The simulations parameters used for the convergence study are detailed in Table 1. Fig. 11 shows the settlement path of the granular sample using the molecular dynamics (MD) as well as the settlement paths calculated with the suggested procedure for different parameters. As a first step, the initial number of cycles calculated before the sequential procedure is taken $k_{\mathrm{i}}=10$ and the length of the estimation interval is taken as $h=10$, while varying $k$. It can be noticed in Fig. 11a that the error between the full molecular dynamics and the obtained results decreases with the number of calculated cycles $k$. Similarly, the effect of the estimation interval length on the accuracy of the procedure can be tested by taking a constant initial number of cycles $k_{\mathrm{i}}=10$ and a constant number of calculated cycles $k=40$. Fig. 11a shows that the error decreases when $h$ decreases, as can be expected. More interestingly, it can be seen that the error stabilizes when the length of the estimation interval tends to 0 .

The presented procedure can be used either in a compaction process or for settlement calculation, as stated in the introduction. If used in a compaction process, the procedure is self-consistent since the objective is to obtain a dense granular sample for other studies. In this case, the parameters $k_{\mathrm{i}}, k$ and $h$ are selected in such a way that the dense state is obtained with the minimum number of iterations. However, in case of settlement calculation, the objective is to study the behavior of granular materials under repeated loading, in terms of residual displacements. Therefore, the accuracy of the procedure has to be established carefully, by choosing the suitable parameters $k_{\mathrm{i}}, k$ and $h$, in order to ensure acceptable results.

\subsection{Application and scope}

The suggested procedure is developed to study the mechanism of long term granular materials settlement under cyclic loading. Experimental studies conducted on granular materials [2-4] showed that the permanent
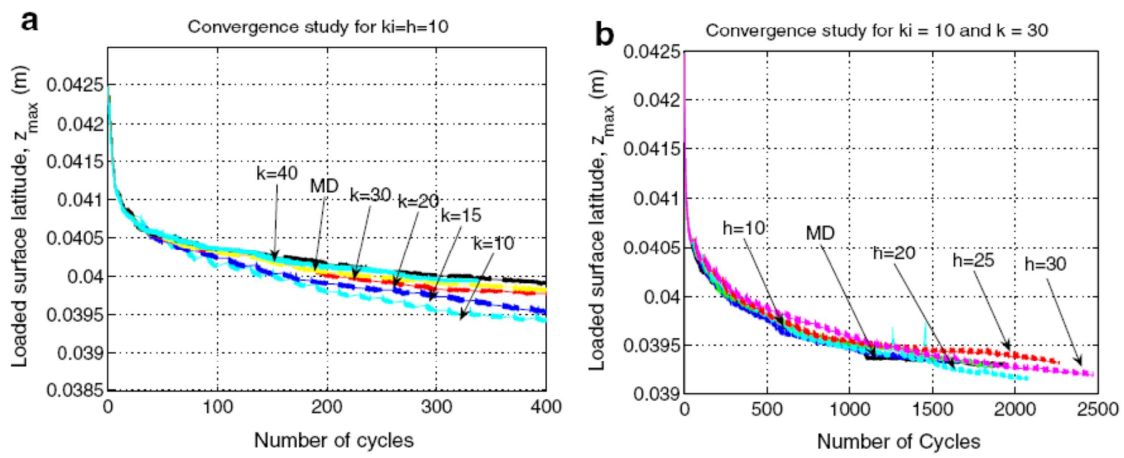

Fig. 11. Convergence of the procedure with the simulation parameters $k$ (a) and $h$ (b) and comparison with the full molecular dynamics calculations. 
displacements depend on several parameters such as the applied force, frequency and material properties. In addition, the mode of loading is expected to have a crucial effect on the amount of settlement when the considered granular sample is periodically excited. In this section, the effect of the gained time on the precision of the whole procedure will be evaluated. Moreover, different modes of loading will be investigated in order to show the limits of the suggested procedure.

The simulations under consideration are performed using the dimensions and material properties presented in Table 1, however, the periodic excitation presented in Fig. 2 will be varied according to the mode of loading. The stress amplitude of the signal applied to the fully confined sample is $\Delta \sigma=14.15 \mathrm{kPa}$ and the excitation frequency is $f=50 \mathrm{~Hz}$. In general, the procedure works properly when the residual displacements are relatively small as compared to the particle size. When the granular material is fully confined the settlement mechanism is mainly related to the particle rearrangement when the excitation take place and consequently, the displacement is relatively small. In this case, it can be seen in Figs. 11 and 12a that the procedure is accurate enough to predict the evolution of the sample with respect to the number of cycles, in terms of residual displacement and coordination number. The precision can also be evaluated in terms of overall residual displacement, coordination number, and configuration as can be seen in Fig. 12. It can be noticed that for all the above mentioned criteria, the relative error increases with the gained time. For instance, it can be noticed that the coordination number presents a relative error of about $12 \%$ when $180 \%$ of gained calculation time is achieved. Moreover, the relative error calculated in terms of configuration precision (using Eq. (22)) is of about $1 \%$ for $180 \%$ of gained calculation time. The residual displacement is evaluated in a more accurate manner since the relative error does not exceed $0.5 \%$ when the same gained time level is achieved.

Unlike the fully confined mode of loading, where the settlement is mainly due to granular materials rearrangement, the partially confined mode of loading results in particles rearrangement as well as particles migration under periodic excitation. In fact, the particles move toward the regions with lower loading level. Therefore, the residual displacement largely undergo the settlement produced in case of equivalent fully confined samples. This behavior makes it difficult to obtain more accurate displacement estimations, thereby inducing anomalous configurations in terms of excessive penetration. For instance, the partially confined loading case of interest in this section is characterized by a stress amplitude of $\Delta \sigma=5.87 \mathrm{kPa}$, an excitation frequency of $f=10 \mathrm{~Hz}$, and a confinement level of $80 \%$.
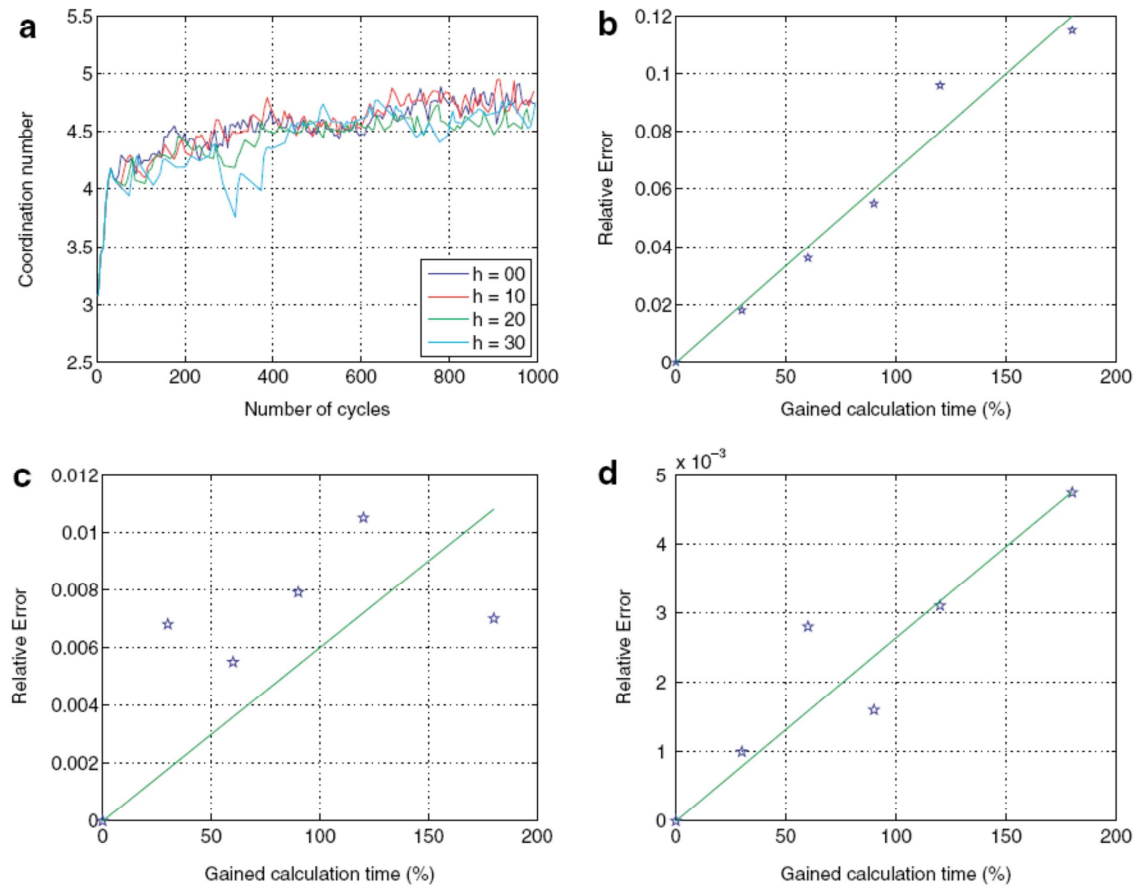

Fig. 12. (a) Variation of the coordination number with respect to the number of cycles, (b) precision in terms of final coordination number, (c) particle positions, and (d) overall residual displacement. 

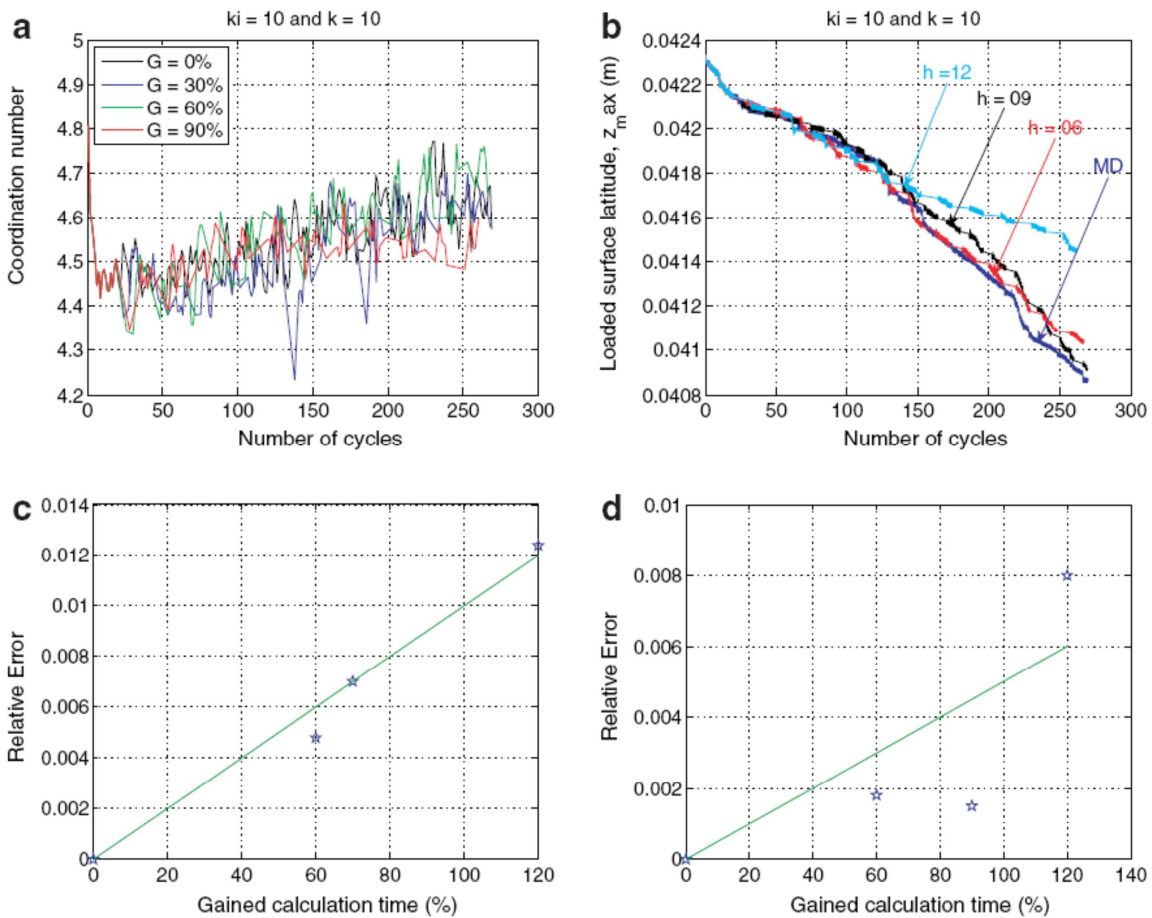

Fig. 13. (a) Coordination number with respect to the number of cycles, (b) Settlement with respect to the number of cycles, relative error in terms of (c) Coordination number and (d) Settlement.

The material properties, sample dimensions, and dissipation parameters are kept unchanged as can be seen in Table 1. Fig. 13a and $\mathrm{b}$ shows the variation of the coordination number and the loaded surface width with respect to the number of cycles. It can be noticed that the slope of settlement is higher in the case of partially confined loading as compared to the fully confined case. However, it can be seen that the coordination number average remains in the same range. On the other hand, Fig. 13c shows the relative error in terms of coordination number with respect to the gained calculation time. Note that the error is calculated relative to the coordination number obtained with full molecular dynamics calculation (since the curve crosses the origin). The same procedure is used to calculate the error in terms of settlement. Fig. 13d shows the variation of error in terms of gained calculation time. It can be noticed that the error in this case is about $8 \%$ when the gained time reaches $120 \%$. This means that the error decreases with the degree of confinement.

The examples presented above demonstrate that the suggested procedure works properly in the case of fully confined modes of loading. However, its accuracy decreases in terms of settlement prediction when it is used for partially confined modes of loading. We also note that in this paper, the procedure is implemented with a molecular dynamics scheme, however, it can be easily adopted in contact dynamic based algorithms.

\section{Conclusion}

A computational procedure for long term granular material settlement calculations has been presented. The suggested procedure sequentially uses the molecular dynamics, a time averaging technique, and a relaxation method in order to estimate the granular flow under cyclic loading. The molecular dynamics represents the engine of the suggested method, it consists of integrating the particle's equations of motion. The Hertz-Mindlin law is used to describe elastic particle interaction, a Coulomb law is used to describe the frictional dissipation, and a proportional damping law is used to describe the viscous aspect of the particles interaction. The averaging technique is based on information collected during the molecular dynamics calculation. A comparison between calculated and estimated configurations has been carried out and showed satisfactory agreements between the averaging and calculated results. Finally, a relaxation method is suggested. It consists of optimizing the granular particles positions in order to determine suitable configurations for further molecular dynamics calculations.

The whole procedure is implemented in an understandable manner in order to calculate granular materials flow under repeated loading. The numerical results from the suggested procedure show that the necessary time for calculating long term settlement is reduced. In addition, the 
suggested procedure provides relatively accurate results. However, according to the convergence study that has been carried out, there is a compromise between the accuracy and the time reduction.

The suggested procedure is mainly developed to study the behavior of granular materials under a large number of repeated cycles. In a future work, it will be applied on several granular samples with different exciting frequencies and degrees of confinement in order to study the mechanism of long term granular settlement.

\section{References}

[1] M. Shenton, Deformation of Railway Ballast under Repeated Loading Conditions, Pergamon, Oxford, 1978.

[2] N. Guérin, K. Sab, P. Moucheront, Identification expérimentale d'une loi de tassement du ballast, Can. Geotech. J. 36 (1999) 523-532.

[3] V.B. Bodin, P. Tamany, K. Sab, P.-E. Gautier, Experimental determination of a settlement of the ballast portion of railway tracks subjected to lateral charging, Can. Geotech. J. 43 (10) (2006) 1028 1041.

[4] A. AlShaer, D. Duhamel, K. Sab, V.H. Nguyen, G. Foret, E. Merliot, L. Schmitt, Dynamic design of a reduced-scale railway test bench using the cesar-lcpe computation code, Bull. Lab. Ponts Chaussées 256-257 (2005) 227-242.

[5] V.H. Nguyen, D. Duhamel, B. Nedjar, A continuum model for granular materials taking into account the no-tension effect, Mech. Mater. 35 (2003) 955-967.

[6] M. Abdelkrim, G. Bonnet, P. De Buhan, A computational procedure for predicting the long term residual settlement of a platform induced by repeated traffic loading, Comput. Geotech. 30 (2003) $463-476$.

[7] G. Saussine, C. Cholet, P.E. Gautier, F. Dubois, C. Bohatier, J.J. Moreau, Modelling ballast behavior under dynamic loading, part 1: A $2 \mathrm{~d}$ polygonal discrete element method approach, Comput. Methods Appl. Mech. Engrg. 195 (19-22) (2005) 2841-2859.

[8] A.S.J. Suiker, E.T. Selig, R. Frenkel, Static and cyclic triaxial testing of ballast and subballast, J. Geotech. Geoenviron. Engrg. 131 (2005) $771-782$.

[9] A.S.J. Suiker, R. de Borst, A numerical model for cyclic deterioration of railway tracks, Int. J. Numer. Methods Engrg. 57 (2003) 441-470

[10] F. Pradel, K. Sab, Homogenization of discrete media, J. Phys. IV 8 (8) (1998) 317-324

[11] P.A. Cundall, O.D.L. Strack, A discrete numerical model for granular assemblies, Géotechnique 29 (1979) 47-65.
[12] J-N. Roux, F. Chevoir, Simulation numérique discrète et comportement mécanique des matériaux granulaires, Bull. Lab. Ponts Chaussées (254) (2005) 109-138.

[13] F. Da Cruz, S. Emam, M. Prochnow, J.-N. Roux, F. Chevoir, Rheophysics of dense granular materials: discrete simulation of plane shear flows, Phys. Rev. E 72 (021309) (2005).

[14] M. Allen, D. Tildesley, Computer Simulation of Liquids, Oxford Science Publications/Oxford University Press, Bristol, 1989.

[15] H. Hertz, Über die berührung fester elastischer körper (on the contact of elastic solids), J. Reine Angew. Math. 92 (1882) 156.

[16] R.D. Mindlin, H. Deresiewicz, Elastic spheres in contact under varying oblique forces, Trans. ASME, Ser. E, J. Appl. Mech. 20 (1953) 327-344.

[17] K.L. Johnson, Contact Mechanics, Cambridge University Press, UK, 1985.

[18] A. Di Renzo, F.P. Di Maio, Comparison of contact-force models for the simulation of collisions in dem-based granular flow codes, Chem. Engrg. Sci. 59 (3) (2004) 525-541.

[19] J.N. Roux, Elasticity, quasistatic deformation, and internal state of sphere packings, in: 17th ASCE Engineering Mechanics Conference, June 13-16 2004, Newark, DE, 2004.

[20] D. Elata, J.G. Berrayman, Contact force-displacement laws and mechanical behaviour of random packs of identical spheres, Mech. Mater. 24 (1996) 229-240.

[21] F. Alonso-Marroquin, H.J. Herrmann, Ratcheting of granular materials, Phys. Rev. Lett. 92 (5) (2004) 54301.

[22] T.G. Sitharam, Discrete element modelling of cyclic behaviour of granular materials, Geotech. Geol. Engrg. 21 (2003) 297-329.

[23] S. Lobo-Guerrero, L.E. Vallejo, Discrete element analysis of railtrack ballast degradation during cyclic loading, Granul. Mat. 8 (2006) 195 204.

[24] G. Ovarlez, E. Clément, Elastic medium confined in a column versus the janssen experiment, Eur. Phys. J. E 16 (2005) 421-438.

[25] S. Alexander, Amorphous solids: their structure, lattice dynamics and elasticity, Phys. Rep. 296 (2-4) (1998) 65-236.

[26] S. Edwards, The equations of stress in a granular material, Physica A 249 (1998) 26-231

[27] A.S.J. Suiker, N.A. Fleck, Frictional collapse of granular assemblies, J. Appl. Mech. (ASME) 71 (2004) 350-358.

[28] G.Y. Onoda, E.G. Liniger, Random loose packings of uniform spheres and the dilatancy onset, Phys. Rev. Lett. 64 (22) (1990) 2727 2730.

[29] N. Bouleau, Probabilité de l'Ingénieur, Variables Aléatoires et Simulation, Editeurs des Sciences et des Arts, Hermann, Paris, 1986.

[30] J. Salençon, Mécanique des Milieux Continus, Editions de l'Ecole Polytechnique, Ellipses, Paris, 2002 